\title{
Le suréquipement des usines hydrauliques
}

\section{Over-equipment of hydroelectric power stations}

\author{
J. Dauzier
}

Contrôleur Général Adjoint

Electricité de France

Direction de la Production et du Transport

Jusqu'en 1962, la production hydraulique et la production thermique ont pris une part égale dans l'alimentation de la France en énergie électrique.

A la fin de l'année 1961, les productions et les puissances des deux sources étaient équilibrées, à savoir : - pour le parc thermique, la puissance était de 10.780 MW et la production annuelle de 38,275 TWh; - pour le parc hydraulique, la puissance était de $11077 \mathrm{MW}$ (dont $6950 \mathrm{MW}$ pour les usines de lacs et d'éclusées) et la production de 38,366 TWh.

C'est alors que l'épuisement progressif des ressources hydrauliques potentielles, le faible coût de la thermie fuel accentuant la tendance, a donné la prépondérance à l'équipement thermique. Au 31 Décembre 1981, pour une puissance totale installée de $71000 \mathrm{MW}$, le système de production français dispose de $51400 \mathrm{MW}$ de puissance thermique (dont $21600 \mathrm{MW}$ nucléaires) et de $19600 \mathrm{MW}$ de puissance hydraulique (dont $11700 \mathrm{MW}$ pour les usines de lacs et d'éclusées). La production hydraulique, en 1981, n'a alimenté qu'un peu plus du quart des besoins du pays, soit 72,4 TWh pour un total de production annuelle de $264 \mathrm{TWh}$.

Mais un rôle nouveau est dévolu à l'énergie hydraulique. Elle s'affirme par ses stations de transfert d'énergie par pompage et aussi par ses usines de lacs et d'éclusées comme productrice d'une énergie de qualité complémentaire de celle du thermique. Ces stations de transfert et ces usines de lacs et d'éclusées sont en effet beaucoup plus aptes que les usines thermiques, classiques aussi bien que nucléaires, à une modulation de la production.

Dans le jeu d'adaptation constante de l'offre à la demande et de maintien de la fréquence et de la tension du réseau, elles ont un rôle essentiel qui est mis en évidence par le graphique de production d'une journée de la saison d'hiver actuelle (fig. 1).

C'est qu'elles peuvent passer de l'arrêt à la pleine puissance en des temps de l'ordre de 5 à 6 minutes. Or,

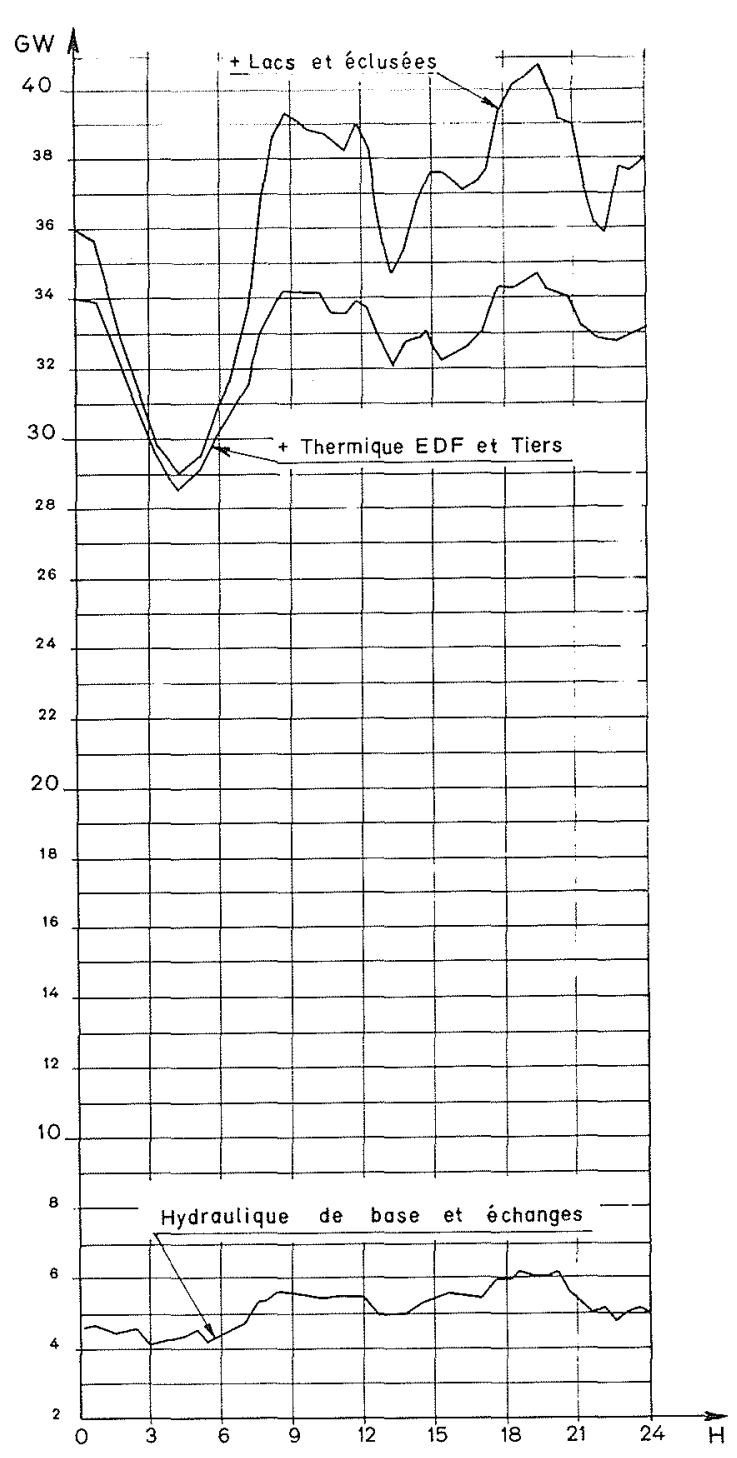

Figure 1. - Puissance consommée le mercredi 25 novembre 1981.

LA HOUILLE BLANCHE/N ${ }^{\circ}$ 5/6-1982 
pour les usines thermiques le passage du minimum technique auquel peut être maintenue une unité thermique ( $1 / 3$ de la puissance) à la pleine puissance requiert, au mieux, 2 à 3 heures et se traduit par une augmentation appréciable du coût du combustible. A celà, il faut ajouter la participation des groupes hydrauliques de lacs et d'éclusées au téléréglage qui équilibre les échanges. Les usines de lacs et d'éclusées y apportent une large contribution, la demi-bande de téléréglage des groupes hydrauliques actuellement équipés pour ce fonctionnement étant de $\pm 1250 \mathrm{MW}$ alors que celle de l'ensemble des groupes thermiques est de $\pm 1700 \mathrm{MW}$. Les indisponibilités des groupes, la satisfaction même des besoins de la consommation, font qu'une fraction seulement de ces possibilités est utilisée chaque jour. La pointe de consommation d'hiver ayant atteint $45000 \mathrm{MW}$, la demi-bande de téléréglage nécessaire au réseau pendant cette période est, en heures de pointe, de l'ordre de $\pm 900 \mathrm{MW}$. En heures creuses d'été, la demi-bande a une valeur de l'ordre de $500 \mathrm{MW}$. Il taut également mentionner la possibilité de marche en compensateur synchrone pour certains groupes hydrauliques ce qui contribue ainsi au maintien de la tension.

La prise en compte de ces qualités dans les calculs économiques, engagés au cours des années qui ont suivi la publication du rapport Pintat en 1976, a fait apparaître l'intérêt de repenser certains sites aménagés et de les suréquiper.

\section{Le programme de suréquipement (fig. 2)}

Nous citerons d'abord deux opérations de suréquipement envisagées dès la construction de l'usine mais dont la conception avait été reportée d'année en année.

\section{Pragnères - Groupe 30,8 $\mathrm{MW}$}

L'usine de Pragnères dans les Pyrénées utilise les eaux accumulées dans les réservoirs de Cap de Long et d'Aubert (capacité utile de $74,9 \mathrm{hm}^{3}$ ), avec un équipement de deux groupes d'une puissance totale de $160 \mathrm{MW}$ turbinant $19 \mathrm{~m}^{3} / \mathrm{s}$ sous 1254 mètres de chute brute. Il s'agit de groupes horizontaux équipés d'un alternateur entre deux roues Pelton en porte-à-faux ; ils ont été mis en service en 1953. La décision d'installer un troisième groupe à l'emplacement qui lui avait été réservé était prise dès 1977.

Ce groupe comportant une seule roue de $30,8 \mathrm{MW}$ de puissance a pu être mis en service à la fin Octobre 1979 et il a porté la puissance de l'usine à $185 \mathrm{MW}$.

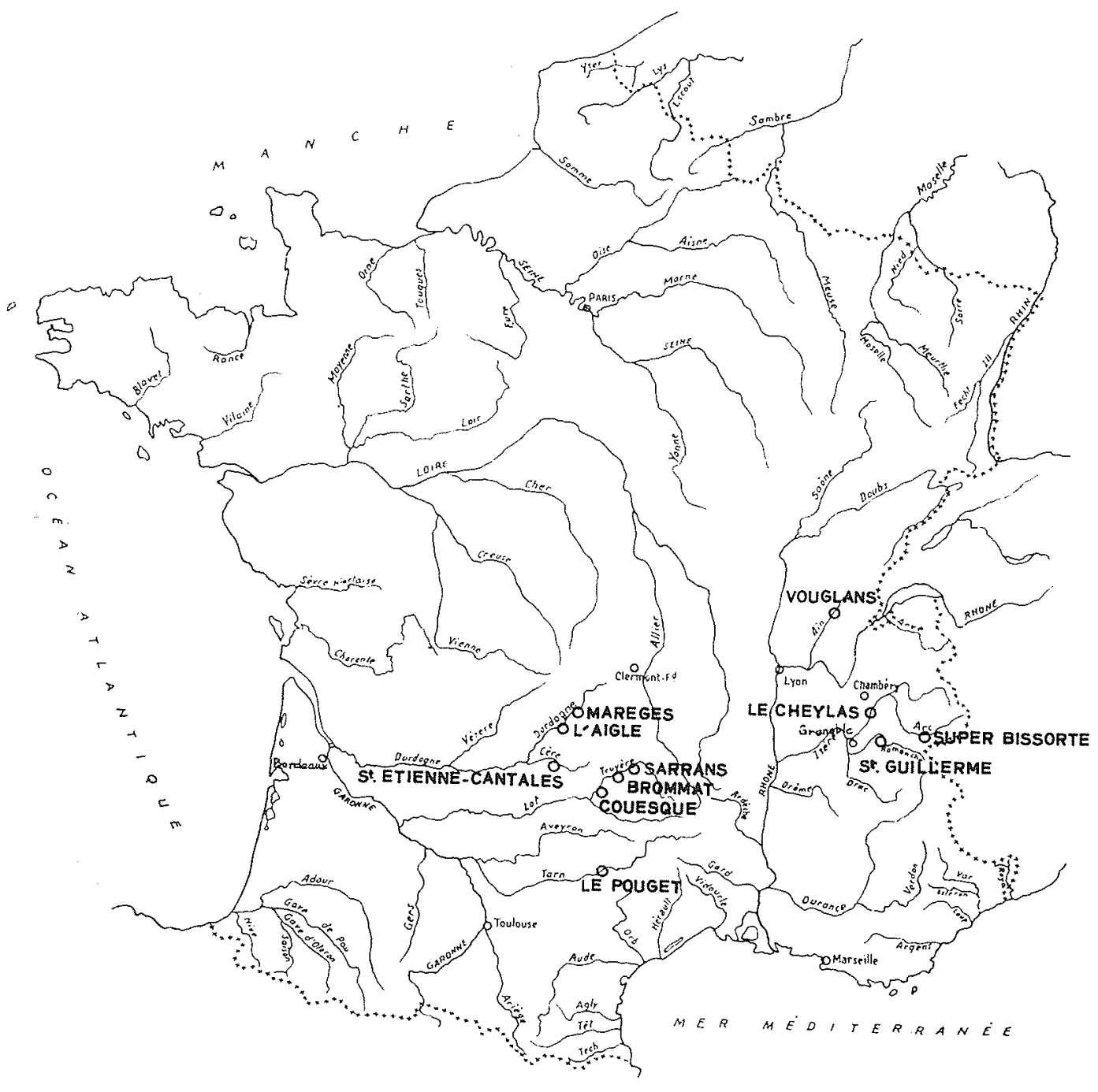

Figure 2. - Usines hydrauliques suréquipées - Situation. 


\section{Saint-Etienne Cantalès - Groupe 38,5 MW}

Accolée au barrage poids voûte qui crée une retenue de $100 \mathrm{hm}^{3(1)}$ sur la Cère, affluent de la rive gauche de la Dordogne, l'usine de Saint-Etienne Cantalès a été conçue pour un équipement à 3 groupes verticaux identiques de $27 \mathrm{MW}$ chacun. A sa mise en service, en 1945, deux groupes seulement avaient été installés, mais la prise d'eau et sa vanne, la conduite au travers du barrage, le béton de première phase de la machine ainsi que le canal de fuite, avaient été prévus pour ce troisième groupe. En 1979, l'installation en était décidée ; les progrès techniques réalisés permettaient de loger dans le même volume un groupe de $38,5 \mathrm{MW}$ qui a été mis en service en Novembre 1981.

Malgré la modicité de la puissance, le troisième groupe ( $3 \mathrm{MW}$ ) de l'usine de Dampjoux sur le Doubs est à citer parmi ces opérations dont les travaux de génie civil ont été des plus réduits.

Dans tous ces cas, les études n'ont été, hormis celles des machines, ni très longues ni très difficiles.

L'intérêt économique des suréquipements d'usines de lacs et d'éclusées a conduit à revoir les équipements de deux vallées du Massif Central, pour lesquels ont été bâties et mises en œuvre des solutions originales. Nous examinerons successivement la vallée de la Truyère et la vallée de la Dordogne.

\section{Vallée de la Truyère}

En tête de la vallée de la Truyère, le réservoir de Grandval accumule $225 \mathrm{hm}^{3}$. Lui fait suite, après le bassin de compensation de Lanau, le lac de Sarrans $\left(255 \mathrm{hm}^{3}\right.$ ) créé par un barrage poids d'une centaine de mètres de hauteur qui fut mis en service en 1934.

L'usine de Sarrans restitue ses eaux dans la retenue de la Barthe $\left(1,8 \mathrm{hm}^{3}\right)$ alimentant l'usine de Brommat. La retenue de Couesque de $19,7 \mathrm{hm}^{3}$ de capacité utile est située à quelques $\mathrm{km}$ en amont du confiuent de la Truyère avec le Lot.

Avant le lancement de l'opération du suréquipement de Sarrans en 1978, la puissance de la chaîne de ces usines était de $700 \mathrm{MW}$ et la production de 1,4 TWh.

\section{Sarrans - Groupe 63,5 MW (Fig. 3)}

Un débit de $157 \mathrm{~m}^{3} / \mathrm{s}$ sous 90 mètres de chute donnait à l'usine de Sarrans une puissance maximale de $114 \mathrm{MW}$, une productibilité annuelle de $250 \mathrm{GWh}$. Cet équipement a été renforcé par l'adjonction, aux 4 groupes verticaux existants, du 5ème groupe de 63,5 MW de puissance. Implanté en puits dans la partie R.D. du bâtiment d'usine réservé à l'origine au décuvage des transformateurs, il absorbe $89 \mathrm{~m}^{3} / \mathrm{s}$.

Son alimentation a été conçue au travers du barrage qui a été percé par une haveuse à tête. La vidange de la retenue en 1979 a été mise à profit pour réaliser la prise d'eau, ensemble métallique partiellement préfabriqué et accroché au parement amont du barrage.

(1) $\left(1 \mathrm{hm}^{3}=10^{6} \mathrm{~m}^{3}\right)$
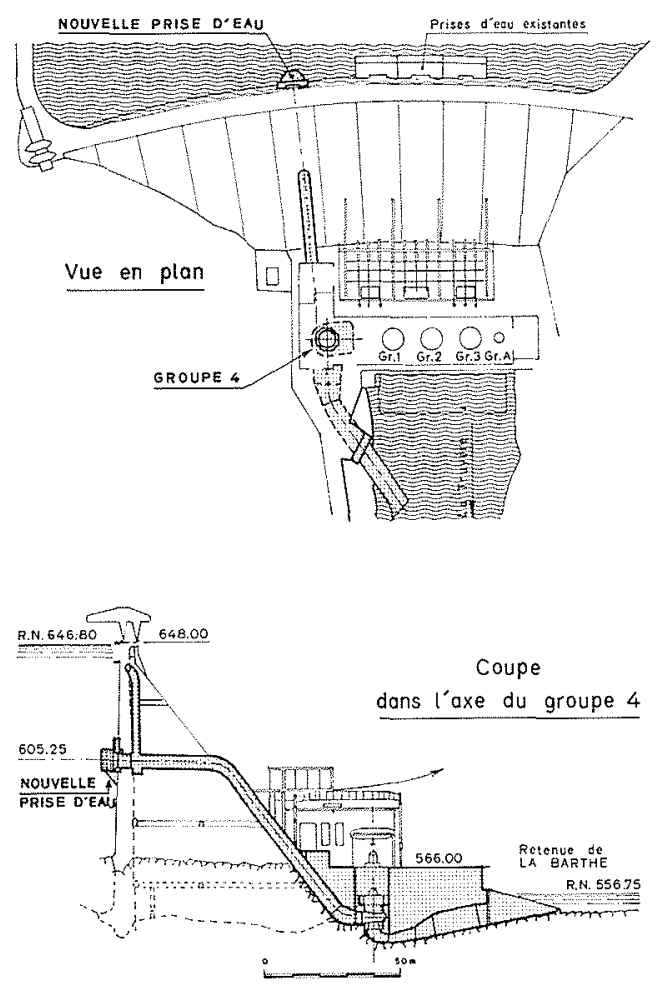

Figure 3.- Suréquipement de Sarrans.

\section{Brommat}

L'usine de Sarrans peut donc turbiner $246 \mathrm{~m}^{3} / \mathrm{s}$. L'usine de Brommat qui lui fait suite prélève ses eaux dans la petite retenue de La Barthe $\left(1,85 \mathrm{hm}^{3}\right)$ et les utilise sous une chute de $260 \mathrm{~m}$; elle a déjà fait l'objet en 1975 d'un suréquipement par la création d'une deuxième usine souterraine abritant un groupe vertical de $242 \mathrm{MW}$, groupe classique le plus puissant actuellement en France. Le renouvellement avec suréquipement de la première usine souterraine n'est pas envisagé dans un proche avenir.

\section{Couesque - Groupe 60,5 MW}

A l'aval de Brommat, le réservoir de Couesque sera le bassin inférieur de la STEP de Montézic ( $888 \mathrm{MW}$ en turbinage $-856 \mathrm{MW}$ en pompage). Sa capacité utile actuelle de $19,7 \mathrm{hm}^{3}$ et les contraintes qu'imposera le fonctionnement de Montézic ont mis en évidence l'intérêt d'un suréquipement de l'usine de Couesque actuellement armée de deux groupes de $33 \mathrm{MW}$. Une unité supplémentaire de $60,5 \mathrm{MW}$, portant le débit turbiné sous la chute maximale de $57 \mathrm{~m}$ à $257 \mathrm{~m}^{3} / \mathrm{s}$, c'est-à-dire le doublant à peu près, sera disposé en puits à l'extrémité de l'usine. L'alimentation utilisera patiellement une dérivation provisoire ancienne prolongée par une galerie nouvelle.

L'opération a été lancée à la fin de 1981 et devrait se terminer à la fin de 1984 .

A signaler que l'aménagement de la vallée pourra être complété à l'aval dans les prochaines années, par 
le suréquipement de l'usine de lac de Lardit (45 MW) qui turbine les eaux de la Selve, affluent de rive gauche de la Truyère $\left(15 \mathrm{~m}^{3} / \mathrm{s}\right.$ sous $352 \mathrm{~m}$ de hauteur de chute brute). Diverses variantes sont à l'étude dont certaines vont jusqu'à tripler la puissance de l'usine.

\section{Vallée de la Dordogne}

En tête de la vallée, le réservoir de Bort crée une retenue saisonnière de $407 \mathrm{hm}^{3}$.

On trouve à l'aval, celui de Marèges $\left(35 \mathrm{hm}^{3}\right)$, celui de l'Aigle $\left(158 \mathrm{hm}^{3}\right)$ enfin, la retenue de Chastang $\left(116 \mathrm{hm}^{3}\right)$ et le bassin de démodulation créé par l'usinebarrage d'Argentat.

L'ensemble représente une puissance de $840 \mathrm{MW}$ et une production de 1,6 TWh. En 1976, EDF s'est penché sur l'équipement de cette vallée du Massif Central, qui est d'un grand intérêt énergétique par le placement d'énergie en période d'hiver, grâce à son important réservoir de tête et grâce aussi au régime climatique dont bénéficie la région.

\section{L'Aigle - Groupe 133 MW - Fig. 4}

Si l'équipement de Chastang dont le $3^{\mathrm{e}}$ groupe a été mis en place en 1958 semble satisfaisant, celui de l'Aigle pourrait être augmenté très au-delà de sa valeur actuelle. Le suréquipement a été fixé à $196 \mathrm{~m}^{3} / \mathrm{s}$, portant le débit turbiné sous une chute de $80 \mathrm{~m}$ à un peu plus de $500 \mathrm{~m}^{3} / \mathrm{s}$. La mise en place de ce groupe supplémentaire de 133 MW a été entreprise en 1979.

L'usine de l'Aigle étant incorporée à son barrage en arc, le projet consistait à créer une galerie indépendante qui contourne l'appui rive droite du barrage. D'un diamètre de $8 \mathrm{~m}$ à l'amont, elle est blindée à $7 \mathrm{~m}$ de diamètre dans sa portion aval protégée par une vanne de tête en puits. Cette adduction de $280 \mathrm{~m}$ de longueur depuis la prise d'eau, alimente une groupe en puits dans un bâtiment d'usine indépendant. L'ensemble a été réalisé avec les sujétions de voisinage des installations en service et avec les précautions que cela requiert. La vidange de la retenue a l'été 1981 a été mise à profit pour faire déboucher la galerie d'adduction dans la retenue et construire la nouvelle prise d'eau. Aux premiers jours de juin 1982, le nouveau groupe $133 \mathrm{MW}$ a débité sur le réseau.

\section{Marèges - Groupe $122 \mathrm{MW}$}

Les études poursuivies sur les deux usines à l'amont de l'Aigle, Bort et Marèges, ont fait proposer un suréquipement de l'usine de Marèges.

Une demande de concession faisant état d'un protocole d'accord EDF - SNCF a été déposé par EDF pour la création d'une nouvelle usine équipée d'un groupe identique à celui de l'Aigle ; cette usine sera implantée en rive gauche à l'aval du barrage dont elle utilisera la retenue.

La conception des nouveaux ouvrages est la même que celle de L'Aigle, l'adduction contournant l'appui rive gauche du barrage-voûte.

Ce groupe de $122 \mathrm{MW}$ de puissance maximale, compte tenu d'une chute un peu plus faible, absorbera
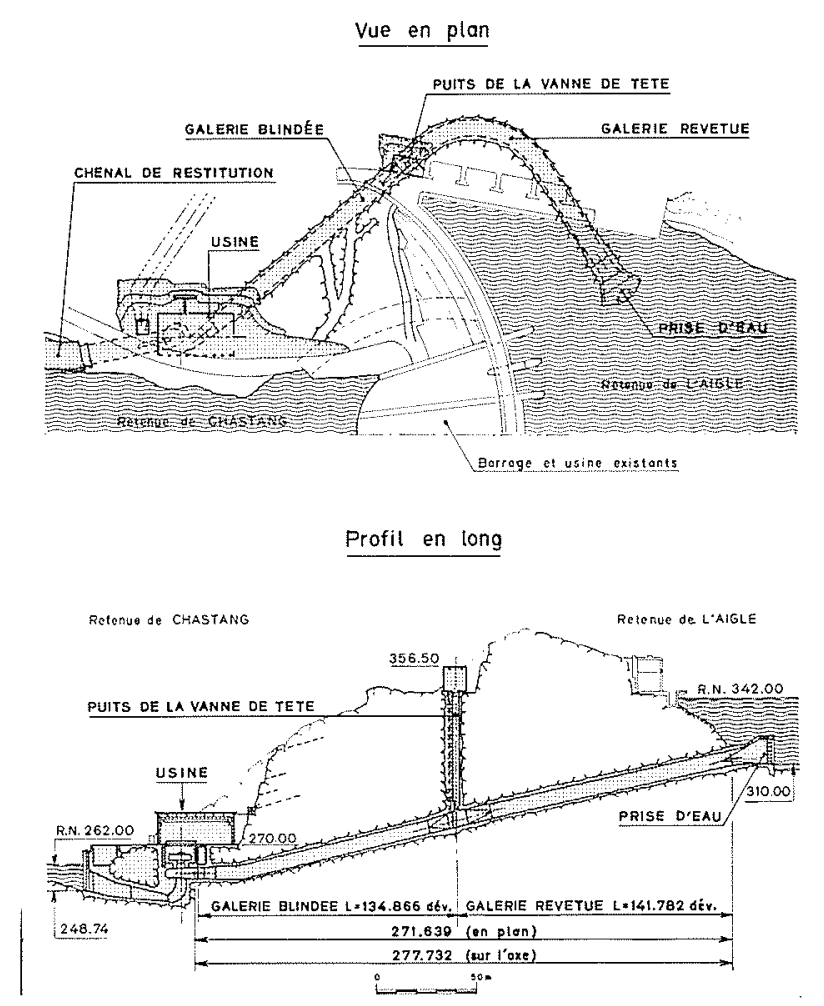

Figure 4.- Suréquipement de L'Aigle.

$190 \mathrm{~m}^{3} / \mathrm{s}$ et donnera à la chute un débit maximal de $430 \mathrm{~m}^{3} / \mathrm{s}$.

\section{Le Pouget - Groupe 257 MW - Fig. 5}

Cependant, l'opération la plus importante engagée par EDF dans ce domaine est la mise en place d'un groupe supplémentaire à l'usine du Pouget. Aux trois groupes Pelton de $40 \mathrm{MW}$ de puissance unitaire, s'ajoutera en effet, à la fin de l'année 1982, un groupe de $257 \mathrm{MW}$. Une telle augmentation de puissance ne pouvant se faire sans une refonte importante des ouvrages, cette adjonction se traduira par la création d'une nouvelle adduction, travaux d'une certaine ampleur.

L'usine du Pouget en rive droite du Tarn turbine en effet sous $461 \mathrm{~m}$ les eaux de la retenue de Villefranche de Panat $\left(8,7 \mathrm{hm}^{3}\right)$ par l'intermédiaire d'une galerie de $4,7 \mathrm{~km}$ de longueur, d'un bassin de faible capacité $\left(655000 \mathrm{~m}^{3}\right)$, le bassin de St-Amans, d'une deuxième galerie de $600 \mathrm{~m}$ et d'une conduite forcée aérienne. Galeries et conduite forcée ont dû être doublées et dimensionnées pour le débit du nouveau groupe $\left(65 \mathrm{~m}^{3} / \mathrm{s}\right)$. Une reprise délicate en sous œuvre du bâtiment du Pouget imposée par l'exiguité du site, a fait placer le groupe $257 \mathrm{MW}$ en puits à l'extrémité de l'usine actuelle. L'alimentation directe de la retenue de Villefranche de Panat par la réserve interannuelle de Pareloup $\left(168 \mathrm{hm}^{3}\right)$ et la position de ce groupe dans le réseau lui donnent un bon intérêt économique qui réside essentiellement dans la production d'énergie d'hiver (puissance garantie produite aux heures critiques et puissance de pointe).

Avant de quitter le Tarn, citons pour mémoire, car la puissance n'est pas à la même échelle, le suréquipe- 


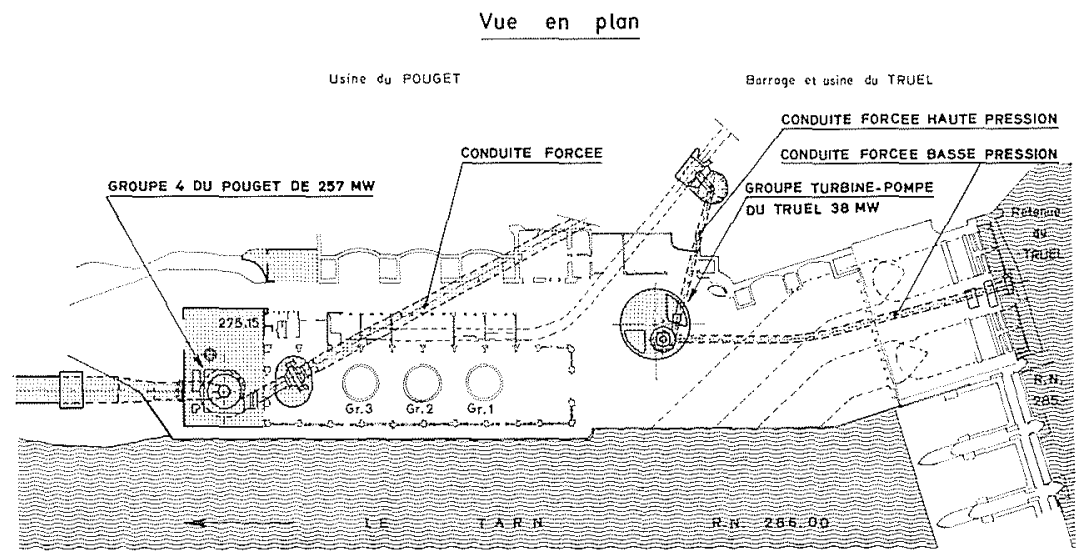

Figure 5.- Suréquipement du Pouget.

ment prochain de l'usine de La Jourdanie, sur le Tarn, barrage-usine de $14 \mathrm{~m}$ de chute où sera installé un groupe supplémentaire de $9 \mathrm{MW}$.

Mais il faut surtout signaler que sur le site même du Pouget, EDF met actuellement en place un groupe réversible, pompe-turbine à deux étages réglables; celui-ci prélèvera les eaux à l'amont du barrage-usine de Truel et améliorera le remplissage des réserves du plateau, Villefranche de Panat et Pareloup, par l'intermédiaire du pompage d'Alrance. Son installation qui vise à l'étude de groupes reversibles de haute chute à distributeurs variables ajoutera également $38 \mathrm{MW}$ à la puissance totale du site.

\section{Renouvellement de St-Guillerme}

Les critères économiques actuels ont évidemment modifié l'optique de renouvellement des installations anciennes et leur rajeunissement peut aller bien souvent au delà du simple remplacement des groupes et des installations électriques qui leur sont liées. Il convient en particulier de signaler le renouvellement, actuellement

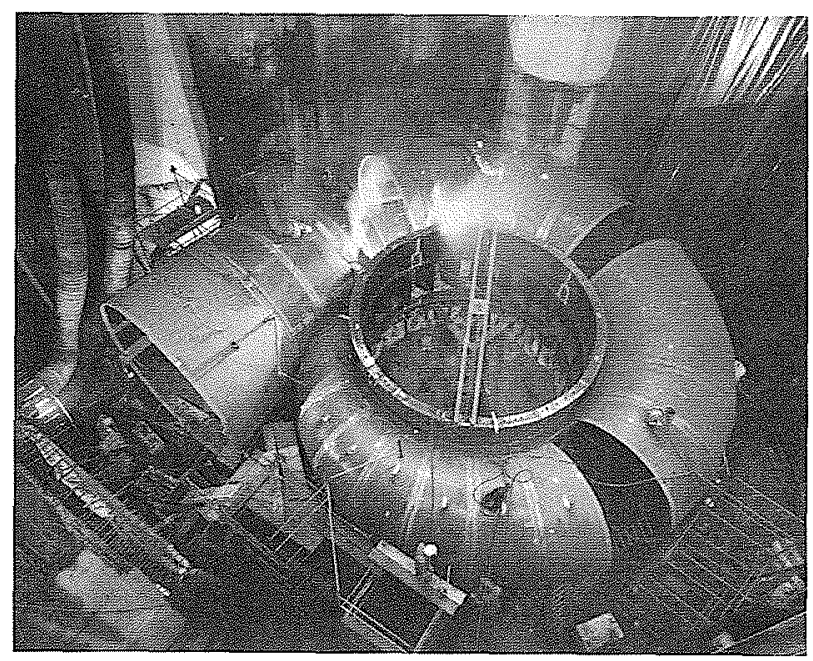

Bâche de L'Aigle en cours de montage. en cours, des usines du Chambon et de St-Guillerme ayant 50 ans d'âge. L'étude s'est finalement orientée vers la réalisation d'une chute unique utilisant la retenue du Chambon $\left(48,5 \mathrm{hm}^{3}\right)$. Nouvelle prise d'eau de lac, galerie en charge de près de $6 \mathrm{~km}$ en rive gauche de la Romanche, puits blindé et usine souterraine équipée de deux groupes Francis d'une puissance totale de $112 \mathrm{MW}$ se sont substitués aux anciens ouvrages de la rive droite. Le gain de puissance est de $89 \mathrm{MW}$; la productibilité de la nouvelle chute est de $214 \mathrm{GWh}$, ce qui correspond à un gain appréciable de $64 \mathrm{GWh}$.

\section{Possibilités de suréquipement en groupes réversibles}

L'ajustement de la production à la consommation aux heures de pointe, ou dans certains cas de défaillance d'unités de production importantes, dans un parc de production où le nucléaire $\mathrm{a}$ un potentiel croissant (21.600 MW de puissance à la fin de 1981 et près de $100 \mathrm{TWh}$ de production pour l'année), peut ajouter quelque intérêt à l'adoption de groupes réversibles pour les suréquipements. Une installation de pompage peut en effet conduire à des puissances très supérieures à celles que l'on installerait pour obtenir un suréquipement classique. Il faut, toutefois, indépendamment de tout problème d'environnement que peut soulever une modulation importante des débits de l'usine, disposer d'un bassin aval.

Quelques exemples d'aménagements en service ou en cours de réalisation sont à citer :

\section{Vouglans}

L'équipement de la chute de Vouglans sur la rivière d'Ain dont la retenue a une capacité utile de $425 \mathrm{hm}^{3}$, turbine $323 \mathrm{~m}^{3} / \mathrm{s}$ sous une hauteur maximale de $97 \mathrm{~m}$. Prévu dès l'origine pour une faible durée d'utilisation, il a vu en 1973 s'accroître cette utilisation par la substitution d'un groupe réversible turbine-pompe de $57 \mathrm{MW}$ au quatrième groupe Francis classique initialement prévu. Pour une puissance installée totale de $264 \mathrm{MW}$ la production est de $270 \mathrm{GWh}$. 


\section{Le Cheylas}

Le même accroissement de la durée d'utilisation a été obtenu sur la chute Arc-Isère du Cheylas par l'adoption, dès la construction, de deux groupes réversibles de $240 \mathrm{MW}$ fonctionnant sous une chute de $261 \mathrm{~m}$ à $245 \mathrm{~m}$. Ils ajoutent aux $675 \mathrm{GWh}$ de production annuelle gravitaire, par le moyen du pompage, $230 \mathrm{GWh}$ supplémentaires en heures pleines et heures de pointe.

\section{Super Bissorte}

L'usine de Bissorte turbine sous une chute de $1159 \mathrm{~m}$, avec une puissance maximale de $75 \mathrm{MW}$, les eaux accumulées dans un réservoir de $39,6 \mathrm{hm}^{3}$ à la cote 2081,80 NGF.

La retenue du Pont des Chèvres $\left(1,2 \mathrm{hm}^{3}\right)$ créée par le barrage du Freney à l'aval de Bissorte et qui alimente l'usine d'Orelle a permis de concevoir un suréquipement important, à savoir :

- Bissorte 2 - Quatre groupes réversibles d'une puissance totale installée en turbine de $600 \mathrm{MW}$;

- Bissorte 3 - Un groupe Pelton de $150 \mathrm{MW}$ $\left(14,5 \mathrm{~m}^{3} / \mathrm{s}\right)$.

La mise en service industriel aura lieu en 1986.

Dans la même optique, nos réflexions se tournent actuellement vers les aménagements de la Durance, mais il est sans doute un peu tôt pour présenter des solutions. Les choix ne pourront être faits pour les deux aménagements de Castillon et Serre-Ponçon qui retiennent actuellement notre attention, qu'après études de simulation.

\section{Castillon}

Concédé en 1928, mis en service en 1948, cet aménagement utilise une réserve de $113 \mathrm{hm}^{3}$, sous une chute maximale de 90 mètres, par 4 turbines Francis de $15 \mathrm{MW}$ absorbant $76 \mathrm{~m}^{3} / \mathrm{s}$ au total.

Un suréquipement qui doublerait la puissance en turbine pourrait se faire par un groupe réversible.

Le fonctionnement en pompe, l'enfoncement de la machine qu'il exigera par rapport au plan d'eau aval, induisent des coûts supplémentaires notamment de génie civil. L'étude économique nous dira s'ils sont compensés par les avantages liés à des possibilités de stockages supplémentaires ou de participation aux problèmes de réglage du réseau.

\section{Serre-Ponçon}

Pour l'usine de Serre-Ponçon, qui peut turbiner $376 \mathrm{~m}^{3} / \mathrm{s}$ sous 128 mètres de chute brute et dont l'équipement avec 4 groupes Francis verticaux a une puissance maximale de $385 \mathrm{MW}$, une étude analogue est à faire et une solution optimale à définir. Elle pourrait conduire à l'adjonction d'un groupe réversible augmentant la puissance en turbine de $160 \mathrm{MW}$. Ce groupe aurait un débit en pompe de l'ordre de $90 \mathrm{~m}^{3} / \mathrm{s}$, ce qui entraînerait une surpuissance aux heures de pointe malgré la différence de débit équipé entre Serre-Ponçon et la chaîne aval.

L'étude en est à son début et au problème signalé ci-dessus de coût supplémentaire d'une solution à groupe réversible, s'ajouteront, comme pour Castillon d'ailleurs et toutes les usines du Sud-Est, les sujétions d'évacuation de l'énergie à partir de cette région surproductrice.

Dans la basse Durance, un suréquipement de l'ordre de $50 \mathrm{~m}^{3} / \mathrm{s}$ des usines de Jouques, Saint-Estève, Malemort, Salon et Saint-Chamas pourrait aussi apporter une puissance supplémentaire d'une centaine de MW.

\section{Bilan des suréquipements en cours et conclu- sions}

Les suréquipements d'aménagements existants comme ceux de Pragnères, St-Etienne Cantalès, Sarrans, Couesque, L'Aigle et Marèges peuvent se réaliser sans impact sensible sur l'environnement. Les adductions nouvelles, les travaux de fondation des nouveaux groupes, le raccordement aux ouvrages de fuite sont cependant des opérations délicates. Le souci qu'a l'exploitation de limiter les pertes d'énergie et de ne compromettre ni la sécurité des ouvrages de génie civil, ni celle des machines en service, impose de rechercher des solutions originales ; nous avons cité le percement du barrage de Sarrans à la haveuse à tête, l'adjonction d'une tour de prise entièrement métallique, le percement des puits de vannes de l'Aigle, Couesque et Marèges au raisedrill.

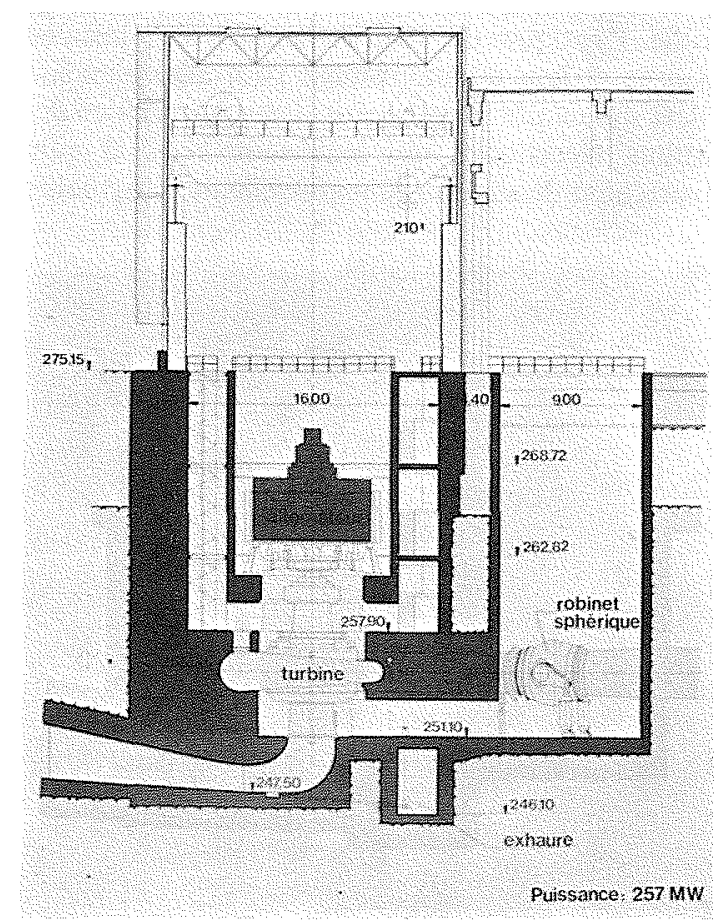

Coupe du groupe de suréquipement 257 MW du Pouget 


\begin{tabular}{|l|c|c|c|c|c|}
\hline \multicolumn{3}{|c|}{ Tableau I. - Caractéristiques des usines après suréquipement } \\
\hline \multicolumn{1}{|c|}{ Aménagement } & $\begin{array}{c}\text { Réserve } \\
\text { utile } \\
\mathrm{hm}^{3}\end{array}$ & $\begin{array}{c}\text { Hauteur } \\
\text { de chute } \\
\mathrm{m}\end{array}$ & $\begin{array}{c}\text { Puissance } \\
\text { MW }\end{array}$ & $\begin{array}{c}\text { Production } \\
\text { GWh }\end{array}$ & $\begin{array}{c}\text { Facteur } \\
\text { d'utilisation } \\
\mathrm{h}\end{array}$ \\
\hline Pragnères & 74,9 & 1.154 & 185 & 170 & 920 \\
St-Etienne Cantalès & 100,6 & 63 & 103,5 & 83 & 800 \\
Sarrans & 255,8 & 90 & 177,5 & 267,5 & 1.510 \\
Brommat & 1,8 & 260 & 412 & 800 & 1,940 \\
Couesque & 19,8 & 57 & 124,5 & 274 & 2.200 \\
L'Aigle & 158,4 & 80 & 341 & 500 & 1.470 \\
Marèges & 35,2 & 78 & 269 & 343 & 1.270 \\
Le Pouget & 177 & 461 & 385 & 278 & 720 \\
\hline
\end{tabular}

\begin{tabular}{|l|c|c|c|c|}
\hline \multicolumn{5}{|c|}{ Tableau II. - Coûts des aménagements } \\
\hline \multicolumn{1}{|c|}{ Aménagement } & Puissance & $\begin{array}{c}\text { Coût } \\
\text { d'engagement }\end{array}$ & $\begin{array}{c}\text { Coût } \\
\text { transposé }\end{array}$ & Coût au kW \\
\hline Pragnères & $30,8 \mathrm{MW}$ & $17 \mathrm{MF}(77)$ & $25,5 \mathrm{MF}(81)$ & $828 \mathrm{~F} / \mathrm{kW}$ \\
St-Etienne Cantales & $38,5 \mathrm{MW}$ & $38 \mathrm{MF}(79)$ & $47,1 \mathrm{MF}(81)$ & $1.220 \mathrm{~F} / \mathrm{kW}$ \\
Sarrans & $63,5 \mathrm{MW}$ & $72 \mathrm{MF}(1 / 78)$ & $103,5 \mathrm{MF}(81)$ & $1.630 \mathrm{~F} / \mathrm{kW}$ \\
L'Aigle & $133 \mathrm{MW}$ & $129,1 \mathrm{MF}(1 / 78)$ & $186 \mathrm{MF}(81)$ & $1.400 \mathrm{~F} / \mathrm{kW}$ \\
Le Pouget & $257 \mathrm{MW}$ & $230 \mathrm{MF}(1 / 78)$ & $331 \mathrm{MF}(81)$ & $1.290 \mathrm{~F} / \mathrm{kW}$ \\
Couesque & $60,5 \mathrm{MW}$ & $111 \mathrm{MF}(81)$ & $111 \mathrm{MF}(81)$ & $1.840 \mathrm{~F} / \mathrm{kW}$ \\
St-Guillerme & $112 \mathrm{MW}$ & $280 \mathrm{MF}(79)$ & $347 \mathrm{MF}(81)$ & $3.100 \mathrm{~F} / \mathrm{kW}$ \\
\hline * Les deux suréquipements de Couesque et St-Guillerme sont également rentabilisés par la production supplémentaire appréciable \\
(64 GWh par an dans l'un et l'autre cas).
\end{tabular}

\begin{tabular}{|c|c|c|c|}
\hline \multicolumn{4}{|c|}{ Tableau III. - Bilan énergétique des suréquipements } \\
\hline Opérations & $\begin{array}{c}\text { Puissance } \\
\text { supplémentaire } \\
\text { MW }\end{array}$ & $\begin{array}{c}\text { Production } \\
\text { supplémentaire } \\
\text { GWh/an }\end{array}$ & $\begin{array}{c}\text { Année de mise } \\
\text { en service }\end{array}$ \\
\hline Pragnères & 25 & 0 & Octobre \\
\hline Sarrans & 63,5 & 17,5 & Août \\
\hline St-Etienne Cantalès & 38,5 & 5 & Novembre 1981 \\
\hline L'Aigle & 133 & 20 & Juin $\quad 1982$ \\
\hline Le Pouget & $25 ?$ & 8 & Octobre \\
\hline St-Guillerme & 89 & 64 & \multirow{2}{*}{$\begin{array}{c}1982-1983 \\
1984\end{array}$} \\
\hline Couesque & 60,5 & 64 & \\
\hline & 666,5 & 178,5 & \\
\hline
\end{tabular}

Dans le domaine des machines, les encombrements ont été réduits par l'adoption de vanne-fourreau à Sarrans, à St-Etienne Cantalès, ̀̀ l'Aigle (vanne de $6,24 \mathrm{~m}$ de diamètre) et à St-Guillerme également. De tels choix ont été générateurs d'une réduction très importante des dépenses de génie civil, du fait des cotes de calage des roues aussi bien à Sarrans qu'a Couesque et à l'Aigle. Pour ce dernier groupe, les dimensions de la fouille du puits-usine, fixées par la fondation de la bâche métallique de $15 \mathrm{~m}$ de diamètre montée sur place, posaient déjà quelques problèmes d'implan- tation dans l'espace réduit, laissé libre, par les ouvrages existants. Un déroctage difficile du pied de versant a cependant été nécessaire.

Les tableaux ci-dessus donnent, pour les centrales suréquipées :

- les caractéristiques des usines ; à leur sujet il convient de signaler que les mesures effectuées récemment sur les nouveaux groupes en service ont confirmé des rendements dépassant $94 \%$, soit un gain de l'ordre de 6 à 8 points par rapport au rendement des machines anciennes. (Tableau I) ; 
- les coûts des aménagements faisant ressortir des coûts au $\mathrm{kW}$ de puissance supplémentaire. (Tableau II); - le bilan énergétique de l'ensemble de ces opérations (Tableau III).

La puissance totale de ces usines, qui participent toutes à la pointe, sera donc augmentée de plus de 660 MW à la fin de l'année 1984. Le suréquipement de Marèges, nouvelle usine de St-Pierre Marèges, dont la demande de concession est en cours d'instruction, portera cette valeur au voisinage de $800 \mathrm{MW}$ à fin 1986.

L'augmentation du prix de la thermie-fuel a donné un intérêt économique particulier à ces augmentations de puissance. Les difficultés préssenties pour le passage des hivers critiques ont incité EDF à en pousser la réalisation qui s'est faite, grâce aux faibles impacts sur l'environnement, dans les meilleurs délais.

Nous avons cité quelques projets en étude qui, sans doute, verront bientôt le jour par la valorisation, dans les calculs économiques, de toutes les qualités de leur énergie. Car la gestion du réseau affirme, nous l'avons dit au début de cet exposé, la valeur des aménagements hydrauliques de modulation. L'énergie hydraulique est une énergie de qualité.

\section{Discussion}

Président : M. DEVOISSELLE

Le Président. - Merci M. DAUZIER pour votre exposé qui nous a montré combien EDF avait su s'adapter et rapidement, à des conditions de marché qui avaient brusquement varié à la suite du choc pétrolier.
J'ai appris aussi que vous aviez utilisé tous les emplacements laissés libres par vos prédécesseurs et que vous en avez même créé de nouveaux. L'exposé de M. DAUZIER était tellement complet je pense qu'il ne peut guère y avoir de question.

\section{Abstract \\ Over-equipment of hydro-electric power stations}

The capacity of the French thermal and hydro-electric parks increased in parallel until the end of 1961, when both had an installed power of $11000 \mathrm{MW}$ and an annual output of $38 \mathrm{TWh}$.

At the close of 1981, hydropower represented only 19600 MW out of a total installed power of $71000 \mathrm{MW}$ and provided a little more than one-fourth of the country's requirements, i.e. 72.4 TWh over $264 \mathrm{TWh}$.

However, hydropower has confirmed its role as producing high-grade energy supplementing thermal generation. The cost of the European thermal unit of fuel oil has led EDF to add to the hydro-electric park by over-equipping lake and lock-gatc plants which provide the supply-demand system with the advantages stemming from the ability of hydroelectric sets to start up rapidly, to modulate their power without substantial financial cost, to reach equilibrium in exchanges and maintain voltage on the system.

Under the programme, commited in 1976 , the installation has been completed of such plants as Praguère in the Pyrenées and St. Etienne Cantales on the Cère, a tributary of the Dordogne in the Massif central. The addition of a further set had been provided for in both cases. The 30.8 MW Pelton set installed at Pragnères and commissioned in 1953 has increased maximum power from $160 \mathrm{MW}$ to $185 \mathrm{MW}$; at St. Etienne Cantales, a vertical $38.5 \mathrm{MW}$ Francis turbine has been added to the two $27 \mathrm{MW}$ generating sets.

New thought has been given to the development of the Truyère and the Dordogne valleys in the Massif Central. The excavation of the weight-dam of Sarrans (height : $100 \mathrm{~m}$ ) on the Truyère which, already harnessed for a capacity of a $700 \mathrm{MW}$ to generated yearly output of $1.4 \mathrm{TWh}$, provided an economical solution and allowed a fifth $63.5 \mathrm{MW}$ Francis turbine to be added to the four generating sets of maximum power $114 \mathrm{MW}$. It was coupled to the system at the beginning of 1981 .

At Couesque (head: $57 \mathrm{~m}$ ) further downstream, an additional $60.5 \mathrm{MW}$ set partly fed by an old penstock will be added to the present two $33 \mathrm{MW}$ sets. It will enter into service in 1984.

The Dordogne valley has a succession of plants at the foot of dams, supplying total power of $840 \mathrm{MW}$ with a yearly output of $1.6 \mathrm{TWh}$.

The over-equipment of the Aigle (head: $80 \mathrm{~m}$ over a $158 \mathrm{~h}$ $\mathrm{m}^{3}$ reservoir) started in 1979 and is now being completed. A $133 \mathrm{MW}$ set will increase the maximum power of the plant to $341 \mathrm{MW}$. It is fed by a gallery by-passing the right bank support of the dam.

Upstream from the Aigle at Marèges, a request for a concession has been made for the installation of a set identical to that of the Aigle. It would be fed, via a new gallery bypassing the left bank support of the arch-dam, by the reservoir presently exploited by the SNCF to operate a $147 \mathrm{MW}$ yower plant.

The largest operation is the installation of a 257 MW set at the Ponget plant on the Tam river. The present plant harnesses the waters of the right bank plateau under a cummulative $460 \mathrm{~m}$ head. The intake structures of the present $40 \mathrm{MW}$ sets have been supplemented with a new gallery (a little over $5 \mathrm{~km}$ long) and a new confined flow conduit. The plant will start to supply the system in Autumn 1983.

It should be noted that the development of the lake-shore and lock plants has allowed an increase in the unit power of plants being renewed. Correspondingly, the St. Guillerme plant will replace the old plants of the Chambon and St. Guillerme and with the two $56 \mathrm{MW}$ Francis turbines will provide additional power of $89 \mathrm{MW}$ to the Romanche river valley. 
Other projects which would use reversible sets are under study.

All these operations can be developed without major consequences for the environment. However, the new intake structures, the operation of merging the new sets and their connection to the discharge structures are all difficult matters. The operator's concern to limit energy losses and not to jeopardize the safety of civil engineering facilities or that of machines in operation necessitates a quest for original approaches. Thus the Sarrans dam was exacavated with an undercutter, an entirely metallic tower was added to the upstream face of the dam for the new Sarrans set and the well shafts at the Aigle, Couesque and Marèges were excavated with a raisedrill. The bulkiness of machines has been reduced by the use of a gate-cylinder at Sarrans, St. Etienne Cantales, Aigle (diameter of the gate: $6.24 \mathrm{~m}$ ) and St. Guillerme. The choices made have also led to reduced civil engineering costs. The energy balance of all these operations is given in the Table below:

\begin{tabular}{|c|c|c|c|c|c|}
\hline Development & $\begin{array}{c}\text { Useful } \\
\text { Capacity } \\
\text { (reservoir) } \\
\mathrm{km}^{3} \\
\end{array}$ & $\begin{array}{c}\text { Head } \\
\text { m }\end{array}$ & $\begin{array}{l}\text { Power } \\
\text { MW }\end{array}$ & $\begin{array}{c}\text { Produc- } \\
\text { tion } \\
\text { GWh }\end{array}$ & $\begin{array}{c}\text { Use } \\
\text { Factor } \\
\text { h }\end{array}$ \\
\hline Pragnères & 74,9 & 1154 & 185 & 170 & 920 \\
\hline St-Etienne Cantales & 100,6 & 63 & 103,5 & 83 & 800 \\
\hline Sarrans & 255,8 & 90 & 177,5 & 267,5 & 1510 \\
\hline Brommat & 1,8 & 260 & 412 & 800 & 1940 \\
\hline Couesque & 19,8 & 57 & 124,5 & 274 & 2200 \\
\hline L'Aigle & 158,4 & 80 & 341 & 500 & 1470 \\
\hline Marèges & 35,2 & 78 & 269 & 343 & 1270 \\
\hline Le Pouget & 177 & 461 & 385 & 278 & 720 \\
\hline
\end{tabular}

The total power of these plants, which all contribute to peak-load generation, will be increased by over $660 \mathrm{MW}$ by end 1984. The over-equipment of Marèges, under the new name "Saint-Pierre Marèges" for which the request for a concession is under advisement will increase the figure to approximately 800 MW by end 1986 . 